\title{
STRUCTURAL MODELS FOR NICKEL ELECTRODE ACTIVE MASS*
}

\author{
B.C. Cornilsen, P.J. Karjala, and P.L. Loyselle \\ Michigan Technological University \\ Houghton, Michigan 49931
}

Raman spectroscopic data allow one to distinguish nickel electrode active mass, alpha- and beta-phase materials. Discharged active mass is not isostructural with beta-Ni(OH) 2 . This is contrary to the generally accepted model for the discharged beta phase of active mass. It is concluded that charged active mass displays a disordered and nonstoichiometric, non-close packed structure of the $R \overline{3} \mathrm{~m}$, $\mathrm{NiOOH}$ structure type. Raman spectral data and $\mathrm{x}$-ray diffraction data are analyzed and shown to be consistent with this structural model.

\section{INTRODUCTION}

Raman spectroscopy has proven to be sensitive to subtle structural changes in nickel electrode active mass and related materials. The experimentally observed Raman and IR spectral selection rules provide unique structural information.

Earlier results showed that discharged active mass is not ordered beta-Ni(OH) 2 (ref. 1). In contrast, the similar selection rules observed for charged and discharged active mass indicated closely related structures (ref. 2). A structure was proposed for active mass which contains $\mathrm{NiO}_{2}$ layers in the the $\mathrm{R} \overline{\mathrm{m}} \mathrm{m}$ space group, usually referred to as an oxyhydroxide-type structure. The layer-layer stacking is non-closed packed ABBCCA. The crystal structure, together with empirical formulae, define the site occupancy. Nickel vacancies are induced by the nickel deficit nonstoichiometry. Vacancies may be occupied by alkali cations and/or excess protons. The excess protons need not be incorporated as molecular water in this model. Ionization of the vacant cation sites forms $\mathrm{Ni}$ (IV) point defects on Ni(III) lattice sites. The nonstoichiometry and dopants control the average nickel oxidation state, and this model explains the maximum values observed empirically.

The presence of point defects increases the disorder of the structure (ref. 2). The nonstoichiometry and point defect content are expected to strongly influence two properties critical to optimum electrochemical performance, proton mobility and electrical conductivity. The enhanced properties for the alpha/gamma cycle are consistent with both a larger vacancy content (calculated on the basis of the $\mathrm{NiOOH}$ lattice) and the observed spectral shifts (ref. 2). Proposed structures must also be consistent with experimental $x$-ray powder diffraction patterns.

In this paper we first summarize Raman structural data which allow one to uniquely distinguish active mass, alpha-, and beta-phase materials. This is followed by a structural analysis of charged active mass. Two key structural models of charged active mass will be discussed in terms of the experimental Raman spectra and $x$-ray powder diffraction patterns. These results show the structure of charged active mass is non-close packed with the NiOOH structure.

* This research has been supported by NASA Lewis Research Center. 
Compounds described in this paper were prepared according to literature methods. Chemical alpha, first precipitate beta-, and recrystallized beta-Ni(OH) 2 were made as described by Barnard et al. (ref. 3). Cathodic alpha was prepared as described by MacArthur (ref 4). The discharged alpha-phase was formed by cycling the cathodic alpha to $-0.2 \mathrm{~V}$ in low $\mathrm{KOH}$ electrolyte, $0.2 \mathrm{M}$, to prevent beta-phase formation (ref. 4). The charged gamma-phase was formed by charging this alpha-phase to $+0.51 \mathrm{~V}$, in $0.2 \mathrm{M} \mathrm{KOH}$. The preparation method of Bode et al. was used to prepare the chemically oxidized, gamma-one material (ref. 5). Potentials were measured and reported with respect to a $\mathrm{Hg} / \mathrm{HgO} / 0.2 \mathrm{M} \mathrm{KOH}$ reference electrode.

\section{RESULTS}

Spectra $a$ and $b$ in figure 1 are typical of cycled active mass. Only two lattice modes are exhibited. No $0-H$ stretches are observed above $3000 \mathrm{~cm}^{-1}$. The charged spectrum is similar to that reported by Jackovitz (ref. 6). This discharged spectrum is reported for the first time. The latter spectrum is different than the spectra of the two beta-phases in figures $1 \mathrm{c}$ and $1 \mathrm{~d}$. Figures $1 \mathrm{e}$ and $1 \mathrm{f}$, of alphaphase materials clearly show that cathodic alpha and chemical alpha differ in structure and that neither is the same as discharged active mass. The spectrum of our discharged phase (fig. 1b) differs from Jackovitz's result (ref. 1). The spectrum he reports is similar to that in figure 1d, although only the $3600 \mathrm{~cm}^{-1} \mathrm{O}-\mathrm{H}$ stretch was shown. The spectrum in fig. 1d is that of a first precipitate betaphase. It is not a well ordered material as seen by comparison with figure lc (ref. 3). The latter is of a recrystallized beta-Ni(OH) 2 . Clearly Jackovitz's spectrum was of a disordered beta, not true beta, and not of discharged active mass.

The Raman (fig. 1a) and IR spectra of the charged active mass and the chemically oxidized material are identical, despite the fact that the $x$-ray pattern of the former material is extensively broadened. This indicates that these two materials are isostructural.

A powder pattern of chemically oxidized material (isostructural with charged active mass) shows qualitative agreement with gamma-type patterns of Glemser and Einerhand and Bode et al. (table I) (ref. 5 and 7). The experimental d-spacings agree well, whereas the intensities vary considerably. A re-analysis of this data in terms of the Bode et al. Cm model (six nickel sites per unit cell) and the $\mathrm{R} \overline{3} \mathrm{~m}$ $(Z=1$, one nickel site per unit cell) model has been carried out. The latter model is consistent with Raman and IR data, the former is not. Calculated values for the $\mathrm{R} 3 \mathrm{~m}$ model show better agreement than the Bode et al. unit cell (table 1). Note several lines are predicted by the Bode model which are not observed. No discrepancy between observed and calculated lines occurs for the $\mathrm{R} \overline{3} \mathrm{~m}$ model. More importantly, the agreement between observed and experimental intensities is much improved for the $\mathrm{R} \frac{\overline{3}}{\mathrm{~m}}$ model.

\section{THEORETICAL ANALYSIS}

Raman spectra for beta-Ni(OH) 2 with the brucite $\left(\mathrm{P}^{\overline{3}} \mathrm{~m} 1-\mathrm{D}_{3} \mathrm{~d}^{3}\right), \mathrm{Mg}(\mathrm{OH})_{2}$, structure are expected to display three lattice modes (below $700^{\mathrm{cm}^{-1}}$ ) and one $\mathrm{O}-\mathrm{H}$ structure are expected to display three $3000 \mathrm{~cm}^{-1}$. Spectrum $1 \mathrm{c}$ of recrystallized beta-Ni(OH) 2 agrees with these predictions. These selection rules were first predicted for brucite by Mitra (ref. 8). It is well known that beta-Ni(OH)$)_{2}$ has the brucite structure (ref. 9 ). 
In this structure, the oxygen atoms are hexagonal close packed, $A B A B$, with nickel atoms in between every other layer.

The second structure of interest is one which exhibits $\mathrm{NiO}_{2}$ layer stacking that is non-close packed (i.e. BB, CC or AA). The simplest space group with this packing contains one formula unit $(\mathrm{Z}=1)$ of $\mathrm{NiO}_{2} \mathrm{H}_{\mathrm{x}}$ with ABBCCA stacking (space group $\mathrm{R} \overline{\mathrm{m}}-\mathrm{D}_{3 \mathrm{~d}}$ ). A factor group analysis for this model structure predicts only two Raman active lattice modes and no $\mathrm{O}-\mathrm{H}$ stretching modes for centric $\mathrm{H}$-atoms positioned in the interlamellar space ( $x$ can be 1 to 2). The Raman spectra of active mass display selection rules which are consistent with this structure.

\section{DISCUSSION}

Figure 1 contains spectra of cycled active mass (charged and discharged), two different beta-phase materials, and two different alpha-phase materials. These six materials each display a different spectrum which is indicative of a different structure. Each of these materials was prepared according to literature methods to represent either active mass or one of the traditional models for active mass.

Spectra $a$ and $b$ in figure 1, typical of active mass, are clearly different than the beta- and alpha-phases, although to various degrees. Discharged active mass (fig. la) and the beta-phases ( $c$ and d) are therefore not isostructural. Chemical alpha, cathodic alpha, and discharged active mass (figs. 1e, if and 1b) also differ in structure. It is apparent that the formation process involves structural transformation from an alpha- or beta-type structure to the active mass structure. Recent in situ spectra are consistent with the ex situ spectra. More detailed structural analyses of the beta-and alpha-materials will appear in forthcoming publications.

An $\overline{\mathrm{R}} \overline{\mathrm{m}}$, $\mathrm{ABBCCA}$ structural model has been proposed for charged active mass (ref. 2). This has been based upon the agreement of the observed Raman selection rules with those predicted for this model. This space group was actually first proposed by Glemser and Einerhand and later interpreted in terms of a larger, $\mathrm{Cm}$ unit cell by Bode et al. (ref. 5 and 7 ).

The charged active mass structure can be confirmed by $x$-ray powder pattern analysis. Table I shows the experimental and calculated powder patterns for a chemicaliy oxidized material which is isostructural with charged active mass based on IR and Raman spectra. The agreement between calculated and observed d-spacings and peak intensities supports this $R \overline{3} \mathrm{~m}$ model. The poor agreement between calculated and observed peak intensities and the extra, unobserved peaks calculated for the $\mathrm{Cm}$, Bode et al. model, do not compare favorably. This together with the fact that the vibrational selection rules for the large $\mathrm{Cm}$ unit cell are not consistent with the observed spectra indicates that the $\mathrm{R} \overline{3} \mathrm{~m}$, NiOOH unit cell is the better structural model.

We refer to this as the "charged active mass structure" because the observed Raman and IR selection rules are identical for both charged gamma (fig. 1a) and charged beta electrochemical materials (ref. 2). The difference between these two materials has been accounted for in terms of this $\mathrm{NiOOH}$ lattice and a point defect containing model (ref. 2). The fundamental difference is in the cation vacancy contents. 
The Raman spectral "fingerprints" allow qualitative distinction of several electrode related materials. Structural differences are apparent in the vibrational spectra of nickel electrode active mass and related phases, which were not detected via other techniques such as x-ray diffraction. These differences show that the structures of active mass and those for traditional alpha and beta phases differ. The structural changes which take place during the formation process are mirrored by these differences.

It is concluded that charged active mass is made up of non-close packed $\mathrm{NiO}_{2}$ layers (ABBCCA). The Raman spectroscopic and $x$-ray powder diffraction data are completely consistent with an NiOOH, single formula unit cell in the $\mathrm{R} \overline{3} \mathrm{~m}-\mathrm{D}_{3 \mathrm{~d}} 5$ space group. This type of stacking encourages the stacking disorder which broadens $x$-ray patterns. This disorder is combined with point defects which result from extensive cation nonstoichiometry to form excessively disordered materials with unique electrochemical properties.

\section{REFERENCES}

1. Loyselle, P. L.; Luehrs, D. C.; and Cornilsen, B. C.: Nickel Electrode Structures, Extended Abstracts of the 168th Electrochemical Society Meeting, Las Vegas NV, October, 16, 1985.

2. Loyselle, P. L.; Karjala, P. J.; and Cornilsen, B. C.: Proc. Symp. on Electrochemical and Thermal Modeling of Battery, Fuel Cell, and Photoenergy Conversion Systems, J. R. Selman and H. C. Maru eds., Electrochemical Society, Pennington, NJ, 1986, pp. 114-121.

3. Barnard, R.; Randel1, C. F.; and Tye, F. L.: Studies Concerning The Ageing of Alpha and Beta $\mathrm{Ni}(\mathrm{OH})_{2}$ in Relation to Nickel-Cadmium Cells. Power Sources 8 , J. Thompson, ed., Academic Press, London, 1981, pp. 401-423.

4. MacArthur, D. M.: The Hydrated Nickel Hydroxide Electrode; Potential Sweep Experiments. J. Electrochem. Soc., Vo1. 117, 1970, pp. 422-426.

5. Bart1, H.; Bode, H.; Sterr, G.; and Witte, J.: Zur Kenntnis der Nickel hydroxidelektrode IV. Kristallstrukturuntersuchung des Hochoxidierten Gammaone Nickelhydroxids. Electrochim. Acta, Vol. 16, 1973, pp. 615-621.

6. Jackovitz, J. F.: The Vibrational Spectra of Nickel Hydroxide and Higher Nickel Oxide. Proc. Symp. on the Nickel Electrode, R.G. Gunther and S. Gross, eds., Electrochemical Society, Pennington NJ, 1982, pp. 48-68.

7. Glemser, 0 , and Einerhand, J.: Die Struktur Hoherer Nickelhydroxyde. 2. Anorg. Allg. Chem., Vol. 261, 1950, pp. 43-51.

8. Mitra, S. S.: Vibration Spectra of Solids. Solid State Physics, F. Seitz and D. Turnbu11, eds., Vo1. 13, 1962, pp. 1-80.

9. Szytula, A.; Murasik, A.; and Balanda, M.: Neutron Diffraction Study of $\mathrm{Ni}(\mathrm{OH}) 2$. Phys. Stat. Sol. B., Vol. 43, 1971, pp. 125-128. 
Calculated and Experimental Powder Patterns for Chemically Oxidized Material Peak Intensities and d-Spacings

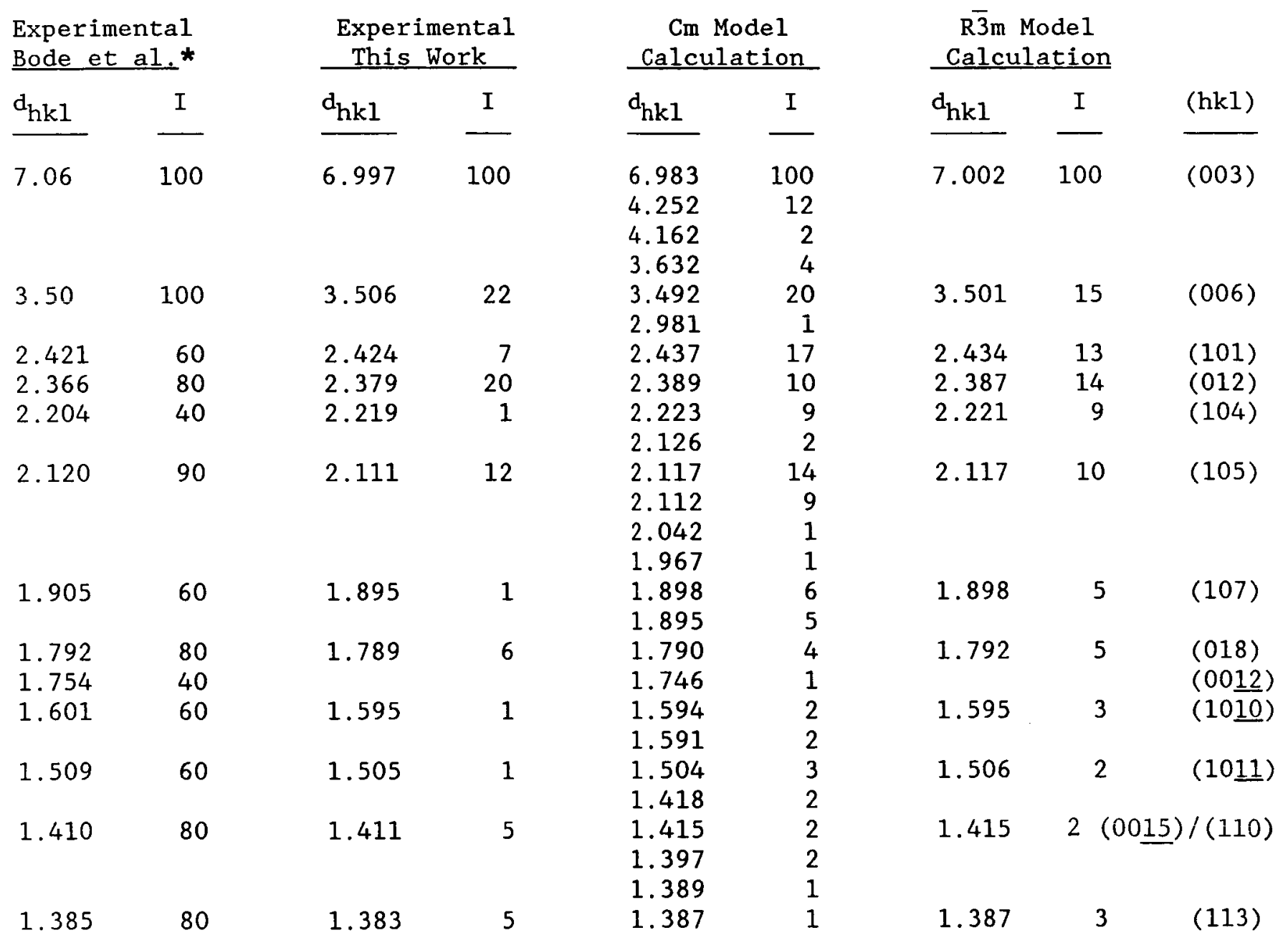

* Joint Committee for Powder Diffraction Standards, Card No. 23-1407. Interplanar spacings, $\mathrm{d}_{\mathrm{hkl}}$, are in Angstroms, and Miller indices are given for the $\mathrm{R} \overline{3} \mathrm{~m}$, hexagonal cell. 
a.



b.

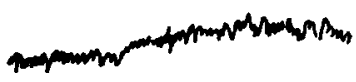

3583

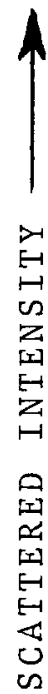
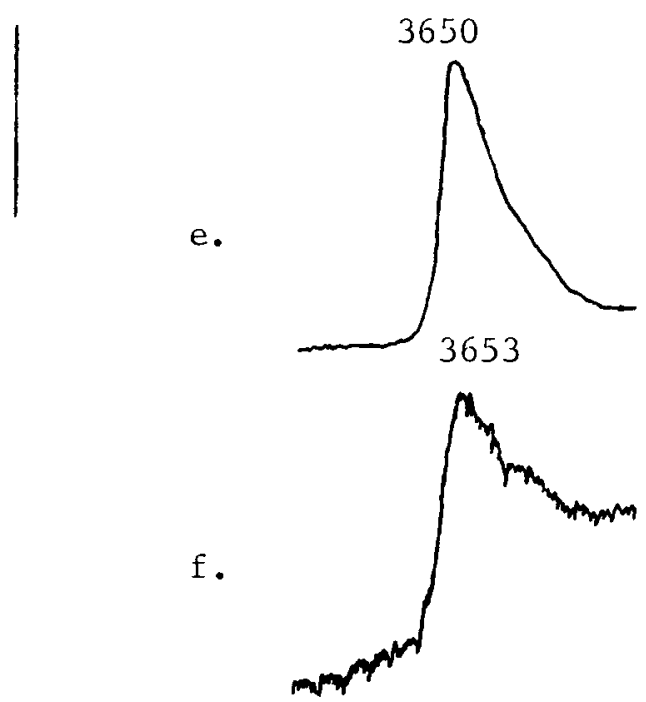
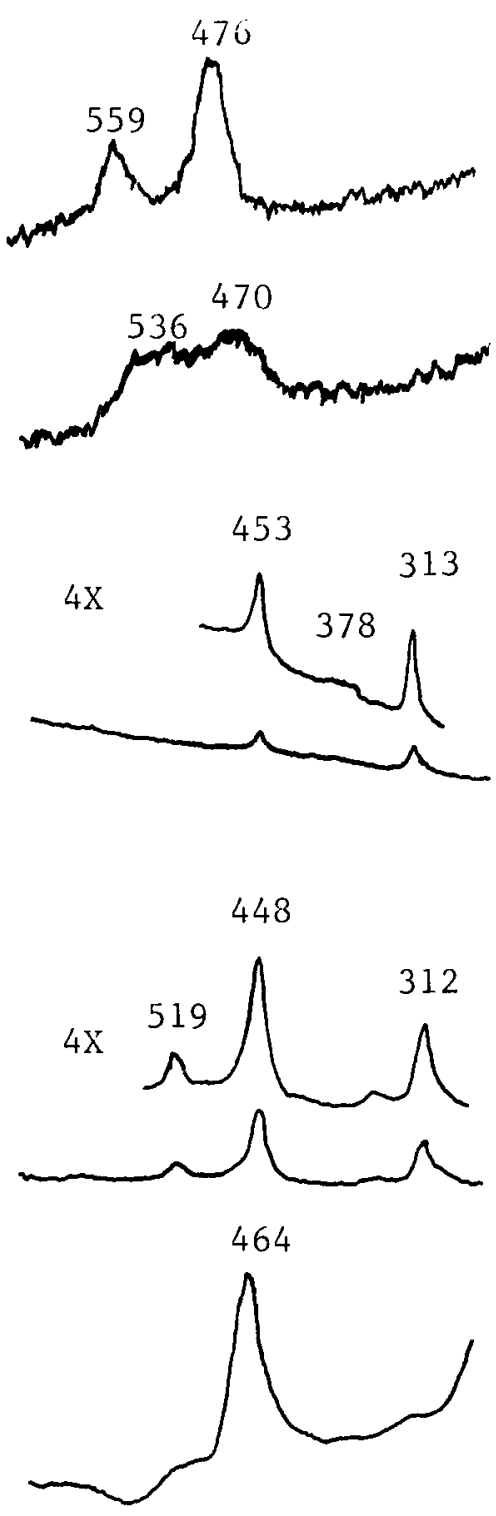

462

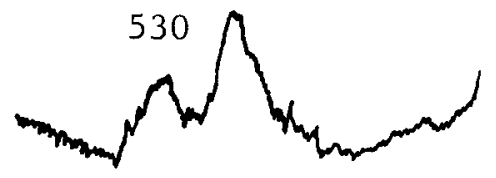

Figure 1. Raman spectra of nickel electrode active mass and model compounds:

a) charged gamma active mass, b) discharged alpha active mass,

c) recrystallized beta-Ni(OH) 2 , d) first precipitate beta-phase,

e) chemical-alpha, and f) cathodic-alpha. 